\title{
HBV Core-Directed Antivirals and Importin $\beta$ Can Synergistically Disrupt Capsids
}

Christine Kim ${ }^{1}$, Christopher Schlicksup ${ }^{1}$, Lauren Barnes ${ }^{1}$, Martin Jarrold ${ }^{1}$, Angela Patterson ${ }^{2}$, Brian Bothner ${ }^{2}$ and Adam Zlotnick ${ }^{1}$

${ }^{1}$ Indiana University, United States, ${ }^{2}$ Montana State University, United States

260 million people suffer from chronic Hepatitis B Virus (HBV) infection, and current therapies are not curative [1]. HAPs are a class of antivirals that have been shown to reduce HBV replication by misdirecting virus assembly [2]. However, HAPs affect other aspects of the HBV lifecycle, but the mechanisms are poorly understood $[2,3]$. HBV core protein $(\mathrm{Cp})$ is involved in almost every stage of the viral lifecycle, including nuclear trafficking [4]. During nuclear transport, HBV capsids bind to host importin $\alpha$ and $\beta$ [5]. The Cterminal domain (CTD) of the $\mathrm{Cp}$, which carries nuclear localization signals and an importin $\beta$-binding sequence, must externalize to the capsid exterior so that capsids can bind to importins $[4,6]$. After binding, the viral cargo is shuttled to the nucleus. Our previous work showed that importin $\beta$ can directly bind to capsids and $\mathrm{Cp}$ in vitro [6]. In a parallel story, the small molecule HAP12 has been shown to stimulate Cp assembly, produce aberrant structures, and bind to HBV capsids [7]. Here we investigated how HAP12-binding influenced the interaction between HBV capsids and importin $\beta$ in vitro. Proteolysis of HAP12-bound empty, pre-genomic RNA, and E. coli RNA-filled capsids showed increased CTD externalization rates. E. coli RNAfilled capsids were more resistant to proteolysis and required higher [HAP12] to increase sensitivity to proteolysis than compared to empty capsids. Transmission electron microscopy (TEM) showed that HAP12bound empty capsids adopted faceted, elongated, and broken structures. Charge detection mass spectrometry (CDMS) revealed that HAP12 increased the amount of importin $\beta$ bound to empty capsids, consistent with increased CTD exposure. Our study also indicated that HAP12 treatment of empty capsids led to the formation of broken capsids and heterogenous importin $\beta$-Cp complexes, both small and large. However, E. coli RNAfilled capsids exhibited neither increased importin $\beta$-binding nor capsid disruption with addition of HAP12 and importin $\beta$. For empty capsids, the formation of disrupted capsids indicates that HAP12 and importin $\beta$ act synergistically to destabilize capsids. Our work showed that core protein-targeted antivirals can be used to perturb viral-host protein interactions. Catalyzing capsid disruption is an unexpected additional mechanism of action for antiviral molecules like HAPs. Untimely capsid disassembly not only can hamper important HBV lifecycle steps, but it can also cause the virus to become vulnerable to host innate immune responses.

\section{References}

[1] Hepatitis B. World Health Organization (2018). at < http://www.who.int/news-room/factsheets/detail/hepatitis-b >.

[2] Feng, S., Gao, L., Han, X., Hu, T., Hu, Y., Liu, H., Thomas, A., Yan, Z., Yang, S., Young, J., Yun, H., Zhu, W. \& Shen, H. Discovery of Small Molecule Therapeutics for Treatment of Chronic HBV Infection. ACS Infectious Diseases 4, 257-277 (2018).

[3] Guo, F., Zhao, Q., Sheraz, M., Cheng, J., Qi, Y., Su, Q., Cuconati, A., Wei, L., Du, Y., Li, W., Chang, J. \& Guo, J. HBV core protein allosteric modulators differentially alter cccDNA biosynthesis from de novo infection and intracellular amplification pathways. PLOS Pathogens 13, e1006658 (2017).

[4] Venkatakrishnan, B. \& Zlotnick, A. The Structural Biology of Hepatitis B Virus: Form and Function. Annual Review of Virology 3, (2015).

[5] Rabe, B., Vlachou, A., Pante, N., Helenius, A. \& Kann, M. Nuclear import of hepatitis B virus capsids and release of the viral genome. Proceedings of the National Academy of Sciences 100, 9849-9854 (2003).

[6] Chen, C., Wang, J., Pierson, E., Keifer, D., Delaleau, M., Gallucci, L., Cazenave, C., Kann, M., Jarrold, M., Zlotnick, A. Importin $\beta$ Can Bind Hepatitis B Virus Core Protein and Empty Core-Like Particles and Induce Structural Changes. PLoS Pathogen 12, e1005802 (2016). 
[7] Bourne, C., Lee, S., Venkataiah, B., Lee, A., Korba, B., Finn, M. \& Zlotnick, A. Small-Molecule Effectors of Hepatitis B Virus Capsid Assembly Give Insight into Virus Life Cycle. Journal of Virology 82, 10262-10270 (2008). 